\title{
Treatment of spondylodiscitis
}

\author{
Kourosh Zarghooni • Marc Röllinghoff • Rolf Sobottke • \\ Peer Eysel
}

Received: 10 October 2011 /Accepted: 9 November 2011 /Published online: 6 December 2011

(C) Springer-Verlag 2011

\begin{abstract}
Purpose Pyogenic infections of the spine are relatively rare with an incidence between 1:100,000 and 1:250,000 per year, but the incidence is increasing due to increases in average lifeexpectancy, risk factors, and medical comorbidities. The mean time in hospital varies from 30 to 57 days and the hospital mortality is reported to be $2-17 \%$. This article presents the relevant literature and our experience of conservative and surgical treatment of pyogenic spondylodiscitis.

Method We have performed a review of the relevant literature and report the results of our own research in the diagnosis and treatment of pyogenic spondylodiscitis. We present a sequential algorithm for identification of the pathogen with blood cultures, CT-guided biopsies and intraoperative tissue samples. Basic treatment principles and indications for surgery and our surgical strategies are discussed.

Results Recent efforts have been directed toward early mobilisation of patients using primary stable surgical techniques that lead to a further reduction of the mortality. Currently our hospital mortality in patients with spondylodiscitis is around $2 \%$. With modern surgical and antibiotic treatment, a relapse of spondylodiscitis is unlikely to occur. In literature the relapse rate of $0-7 \%$ has been recorded.
\end{abstract}

K. Zarghooni $(\bowtie)$

Department of Orthopaedic and Trauma Surgery,

ZKS (BMBF 01KN1106), University of Cologne,

Cologne, Germany

e-mail: kourosh.zarghooni@uk-koeln.de

M. Röllinghoff $\cdot$ R. Sobottke $\cdot$ P. Eysel

Department of Orthopaedic and Trauma Surgery,

University of Cologne,

Cologne, Germany

M. Röllinghoff

Department of Orthopaedic and Trauma Surgery,

Martin Luther University Halle-Wittenberg,

Halle, Germany
Overall the quality of life seems to be more favourable in patients following surgical treatment of spondylodiscitis.

Conclusion With close clinical and radiological monitoring of patients with spondylodiscitis, conservative and surgical therapies have become more successful. When indicated, surgical stabilisation of the infected segments is mandatory for control of the disease and immediate mobilisation of the patients.

\section{Introduction}

Spondylodiscitis is uncommon and, because initial signs and symptoms are nonspecific in nature, a delay before diagnosis and treatment often occurs. In the literature, the average duration between the first symptoms and diagnosis has been reported to be between two and six months [1-8].

The term "spondylodiscitis" refers to the primary infection of the intervertebral disc by a pathogen and secondary osteomyelitis of the adjacent end-plates, usually occurring in conjunction with one another. The incidence of spondylodiscitis varies in developed countries between $1: 100,000$ and $1: 250,000[1,2,9-11]$. It is the main manifestation of haematogenous osteomyelitis in patients aged over 50 years and represents around $3-5 \%$ of all cases of osteomyelitis [12].

In this article we will discuss conservative and surgical treatment and outcomes of pyogenic spondylodiscitis, based on a review of the literature and our own research experience.

\section{General management of spondylodiscitis}

The principles of treatment are eradication of the underlying infection, restoration and preservation of spinal structure and stability, recovery from any neurological deficits, and appropriate pain therapy. 
Immobilisation or fixation of the affected spine segments, antibiotic therapy, and, depending on the extent of the disease, debridement and decompression of the spinal canal are basic requirements for successful treatment yielding complete recovery from spondylodiscitis [12].

Heterogeneity of the patient population as well as variations in treatment complicates the establishment of standard therapeutic guidelines. To date, there have been no randomised controlled trials to guide selection of the appropriate route, duration, and agents for antibiotic therapy [13]. The level of evidence for treatment recommendations does not exceed level C [14].

Microbiological diagnosis is essential to enable targeted antibiotic treatment. Agent-sensitive intravenous antibiotics should be begun preferably only once the pathogen has been identified and a table of resistance/sensitivity has been formulated. Specimens for microbial studies should be taken from the port of entry and at least three sets of blood cultures should be collected at different times after discontinuation of antipyretic and antibiotic agents. If the patient requires urgent treatment due to sepsis or a fulminant disease course, empirical therapy with a broadspectrum antibiotic regimen appropriate to treat the most common pathogens for spondylodiscitis, i.e. Staphylococcus aureus and Escherichia coli, should be initiated only after collecting blood cultures [12]. Based on our experience, we suggest the following algorithm for collection of blood cultures, CT-guided biopsy, and intraoperative sampling (Fig. 1) [12, 15].

The literature offers no standardised guidelines as to the duration of intravenous antibiotic treatment. As a general rule, it is advisable to administer antibiotics intravenously for at least two to four weeks to improve bioavailability. Observational studies have yielded a higher incidence of treatment failure when parenteral therapy was administered for less than four weeks [16, 17]. In individual cases, the switch from parenteral to oral administration of antibiotics can be performed earlier, as soon as the patient's general condition has been reliably stabilised and the serum inflammatory markers have returned to normal or at least significantly improved [12]. The transfer to oral administration can also take place sooner if enteral bioavailability of the active ingredient is high, e.g. as is the case with fluoroquinolones, clindamycin, or linezolid. Linezolid is mainly used in cases of MRSA infection, although one should be aware of the haematopoietic side effects $[6,18,19]$.

Consistent recommendations concerning the overall duration of antibiotic therapy are also lacking in the literature. With regard to the treatment of nonspecific spondylodiscitis, antibiotic administration from six weeks to three months has been suggested [1, 5, 10, 20, 21]. In any case, the duration of treatment depends on the condition of the individual patient. If in doubt, high-risk patients (e.g. those with immunosuppression, diabetes mellitus, drug abuse) in particular should be treated for longer. It is our practice to administer antibiotic therapy until inflammatory parameters have remained within the normal range for six consecutive weeks.

If, however, the historical, clinical, and radiological data suggest tuberculous spondylodiscitis, appropriate therapeutic agents may be initiated. Usually these cases are not fulminant, so that the microbiological findings can be awaited. Based on experience and retrospective data, antituberculous therapy should be continued for at least 18-24 months to allow for complete healing and prevent relapse [12]. Atypical mycobacteria pose a serious therapeutic problem because of frequent resistance [22, 23]. A definitive therapeutic regime for the treatment of atypical mycobacteria has not yet been established. The guidelines of the American Thoracic Society, dating from 1997, recommend combination therapy of isoniazid, rifampicin, and
Fig. 1 Algorithm for the diagnosis and treatment of spondylodiscitis

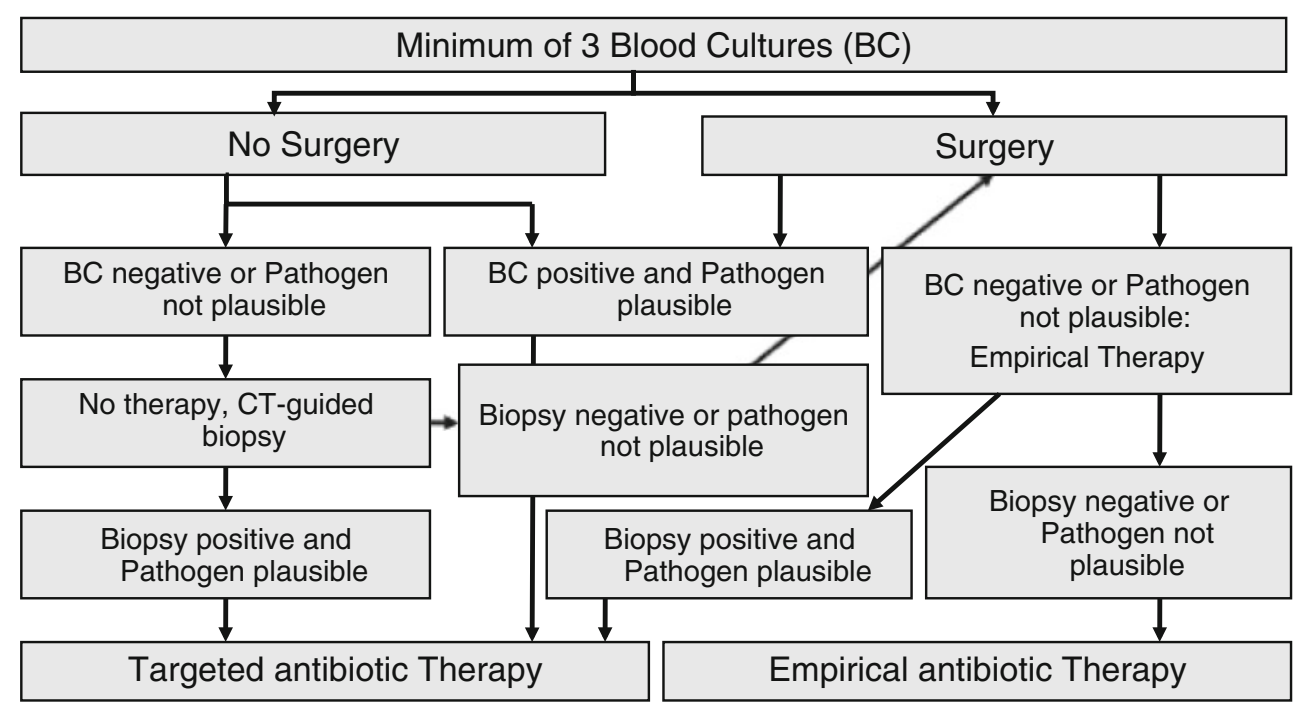


ethambutol, with or without streptomycin or clarithromycin, for the treatment of pulmonary infections [24].

If a mycotic infection has been verified, adequate antimycotic therapy must be initiated. Spondylodiscitis caused by fungi can closely resemble tuberculous spondylodiscitis on magnetic resonance imaging [25]. Overall, it is difficult to identify fungi as a cause for spondylodiscitis, and antimycotic therapy is often complicated. Thus, van Ooij et al. advocate surgical treatment at an early stage of the disease [26].

\section{Conservative treatment}

In the absence of an absolute indication for surgery, when clinical symptoms are mild or bony destruction is minimal, and/or the risks of surgical intervention seem too high, a conservative approach may be considered [3, 7, 12]. With the surgical risks in mind, conservative therapy often is the primary option for elderly patients and for patients in poor general condition [27].

Spinal immobilisation is a very important aspect of treatment, and often presents a challenge in conservative therapy. Adequate immobilisation of the affected segments obviates the need for prolonged bed rest. For the cervical spine, immobilisation can be achieved using a collar or halo-fixator. For the mid-thoracic spine, a reclining brace can suffice. This orthesis holds the affected spine segments in a reclining position, distributing weight to the generally unaffected facet joints, and reducing stress on the diseased vertebra. Even when the thoracolumbar or lumbar region is involved, and destruction is not too severe, mobilisation in a thoracolumbar or lumbosacral orthosis can be considered [12]. However, bed rest for a period of at least six weeks is still required for substantial defects of the anterior column as well as disease affecting the lower lumbar or lumbosacral segments $[3,12]$.

In a retrospective case series analysis, for patients lacking indications for surgical therapy, Flamme et al. recommended conservative therapy with consistent immobilisation of the patient in a reclining plaster shell for six weeks, followed by another six weeks of brace treatment. The plaster shell generated a kyphotic position, and thus achieved fast pain relief [28]. When considering the treatment of the elderly in particular, one must also recognise the well-known morbidity related to bed rest, e.g. decubitus ulceration, deep vein thrombosis, pulmonary embolism, and pneumonia.

In addition to the risks of bed rest, the rates of pseudarthrosis and instability, which can both ultimately result in kyphotic deformation and chronic pain syndromes, are comparatively high at $16-50 \%[2,3,10,28,29]$. Conservative therapy should not be continued past four to six weeks if no radiological evidence of reactive bony fusion is present, if destruction has progressed, or if clinical improvement has not occurred $[3,7,30]$. Thus, we advocate bed rest until pain symptoms are controlled, but generally no longer than two weeks, with subsequent mobilisation in an orthesis.

Within the context of conservative treatment, paravertebral abscess formations requiring decompression can be treated by CT-guided drain insertion and drainage until resorption has been documented by CT-imaging.

\section{Surgical treatment}

Urgent surgical intervention for spondylodiscitis is required when either neurological deficits or sepsis develop. Further indications for surgery are listed in Table 1. Relative indications for surgical treatment include uncontrollable pain symptoms and lack of patient compliance with conservative therapy $[2,9,12,21]$. The goals of surgery are debridement and removal of the septic focus, collection of specimens for microbiological testing and histopathological examination, decompression of the spinal canal, with stabilisation and restoration of the infected spine segment, and subsequent bony fusion. In comparison to conservative therapy, this approach allows for a safer and more rapid cure of the inflammation. Also, mobilisation can be begun shortly after surgery $[3,12,30]$. Since the mid-1950s, when Hodgson et al. published their articles on the treatment of tuberculous spondylodiscitis of the whole spine, anterior debridement with interbody bone grafting became the gold standard therapy, and remained so until the 1990s [31]. However, anterior spinal fusion with bone graft alone does not result in primary stability. It necessitates several weeks of patient immobilisation followed by several months of brace treatment before firm bony union with the graft can be achieved [3]. In contrast to conservative therapy, the fusion rate increased to $90-100 \%[3,32]$. In cases with multi-segmental

Table 1 Indications for surgery in spondylodiscitis

Indications for surgery in spondylodiscitis

1. Neurological deficits

2. Sepsis

3. Significant bone involvement with instability

4. Impending or current deformities

5. Intraspinal space-occupying processes (i.e. spinal abscess)

6. Unclear aetiology of the process and/or suspected malignant disease

7. Failure to respond to conservative therapy

8. Uncontrollable pain

9. Patient's lack of compliance 
involvement and longer fusion spans, however, there are higher rates of complications such as pseudarthrosis and bone graft displacement with subsequent kyphotic deformation [3]. Thus, instrumented stabilisation in conjunction with debridement and graft interposition has been established as the current standard procedure. This enables rapid postoperative mobilisation of the patient and reduces the risks of pseudarthrosis and kyphotic deformation [2, 3, 12].

The intact posterior elements of the affected spine segment should not be destabilised by decompression laminectomy alone, as this may result in further instability and potential deterioration of the neurological situation $[2$, $5,16,33,34]$.

Of course, implantation of fixation materials into an infected site bears the risks of pathogenic colonisation and persistent infection. Spinal instrumentation can be applied successfully, however, if thorough debridement of the infected bone and wound area is provided with concomitant application of local antibiotic agents [10, 12]. Thorough debridement down to the vital, well-perfused spongy bone is essential to enable complete cure of the infection [3, 11, 29]. In common with most spine surgeons we advocate the use of titanium implants, as they do not seem to be associated with increased recurrence rates [4, 35].

Recommendations regarding the optimal surgical strategy for spondylodiscitis remain controversial $[1,2,4,5,7,10,21$, $36,37]$. This is most likely due to patient heterogeneity and variation in therapeutic methods. Surgical therapy for spondylodiscitis can be performed as either one-stage or two-stage surgery. When neurological deficits are present, urgent decompression plus stabilisation should be performed, because the prognosis of neurological sequelae depends on swift surgical intervention [7]. In patients lacking neurological symptoms, a two-stage surgical procedure may be favoured if the patient's general health is reduced. The second stage is performed one to two weeks after the first, depending on the patient's recovery.

Recommendations regarding the choice of interior stabilisation are equally diverse; suggested approaches include purely posterior stabilisation, anterior only, combined posterior-anterior, or combined anterior-posterior stabilisation $[2,3,5,7,10,21,29,36,37]$.

Table 2 presents our current treatment concept of pyogenic spondylodiscitis which is based on the grade of destruction of the affected segments. In our experience the grade of segmental destruction, the necessity for debridement, the segmental kyphosis and the intraspinal extent of the disease are pivotal for the further treatment. Patients with severe destruction or multisegmental disease may need several revisions in sequence for debridement and stabilisation.

In the cervical spine (Fig. 2), an anterior approach can be performed including adequate debridement and stabilisation with structural autologous bone graft (i.e. iliac crest) or a fusion cage filled with autologous trabecular bone graft, along with anterior plate insertion. Locking plate systems may also be implemented, especially when more than one spine segment is diseased (Fig. 3) [5]. Bi- or polysegmental infections may require posterior-anterior stabilisation. Postoperatively, the patient should wear a semi-rigid cervical collar for four to six weeks [38].

When the thoracic spine is affected, a single-step posterior approach may suffice even when there is significant destruction of the anterior column. Stability is generally maintained by the rib cage, and mobility is restricted physiologically [39, 40]. However, anterior instrumentation alone may also be sufficient. Anterior stabilisation of the spine can be achieved either by open transthoracic or open posterolateral approach, or via
Table 2 Conservative and surgical treatment of pyogenic spondylodiscitis. All implants used should be titanium. In very severe spondylodiscitis multiple revisions and debridements might be indicated

\begin{tabular}{|c|c|c|c|}
\hline \multirow[t]{2}{*}{ Segment } & \multicolumn{3}{|l|}{ Grade of destruction } \\
\hline & Low & Moderate & Severe \\
\hline Cervical spine & $\begin{array}{l}\text { 1. Cervical brace } \\
\text { 2. Anterior autologous iliac } \\
\text { bone graft and (locking) plate }\end{array}$ & $\begin{array}{l}\text { 1. Anterior } \\
\text { autologous iliac } \\
\text { bone graft and } \\
\text { (locking) plate }\end{array}$ & $\begin{array}{l}\text { 1. Titanium fusion cage with } \\
\text { autologous cancellous bone } \\
\text { 2. If very severe: Additional } \\
\text { posterior screw-rod-system }\end{array}$ \\
\hline Thoracic spine & $\begin{array}{l}\text { 1. Posterior stabilisation with } \\
\text { percutaneous screw-rod- } \\
\text { system (minimal invasive } \\
\text { spine surgery; MISS) }\end{array}$ & $\begin{array}{l}\text { 1. Thoracoscopic } \\
\text { (up to L2) with } \\
\text { iliac autologous } \\
\text { bone graft and } \\
\text { plate }\end{array}$ & $\begin{array}{l}\text { 1. Transthoracic with } \\
\text { autologous iliac bone graft; } \\
\text { if multisegmental with rib/ } \\
\text { fibula and plate } \\
\text { 2. If very severe: Additional } \\
\text { posterior screw-rod-system }\end{array}$ \\
\hline $\begin{array}{l}\text { Thoracolumbar } \\
\text { and lumbar } \\
\text { spine }\end{array}$ & $\begin{array}{l}\text { 1. Reclining rigid brace } \\
\text { (usually polyethylene) } \\
\text { 2. Posterior stabilisation with } \\
\text { percutaneous screw-rod- } \\
\text { system (MISS) }\end{array}$ & $\begin{array}{l}\text { 1. Posterior lumbar } \\
\text { interbody fusion } \\
\text { (PLIF) with } \\
\text { titanium cage }\end{array}$ & $\begin{array}{l}\text { 1. 1- or 2-step } 360^{\circ} \text { fusion } \\
\text { with anterior iliac bone } \\
\text { graft; if multisegmental } \\
\text { with fibula and posterior } \\
\text { screw-rod-system }\end{array}$ \\
\hline
\end{tabular}


Fig. 2 a T1-weighted sagittal image without gadolinium shows decreased signal intensity from the $\mathrm{C} 3$ and $\mathrm{C} 4$ vertebrae and an epidural collection extending from $\mathrm{C} 2$ to the mid-aspect of C5. b

T1-weighted sagittal image after gadolinium shows significant enhancement of the epidural space at $\mathrm{C} 2-\mathrm{C} 7$ levels and the anterior soft tissues $\mathrm{C} 2-\mathrm{C} 6$. c T2-weighted sagittal image shows increased signal intensity from $\mathrm{C} 3$ and $\mathrm{C} 4$ vertebrae, associated with increased signal from the intervertebral disc $\mathrm{C} 3 / 4$ and evidence of an epidural collection $\mathrm{C} 3-\mathrm{C} 6$

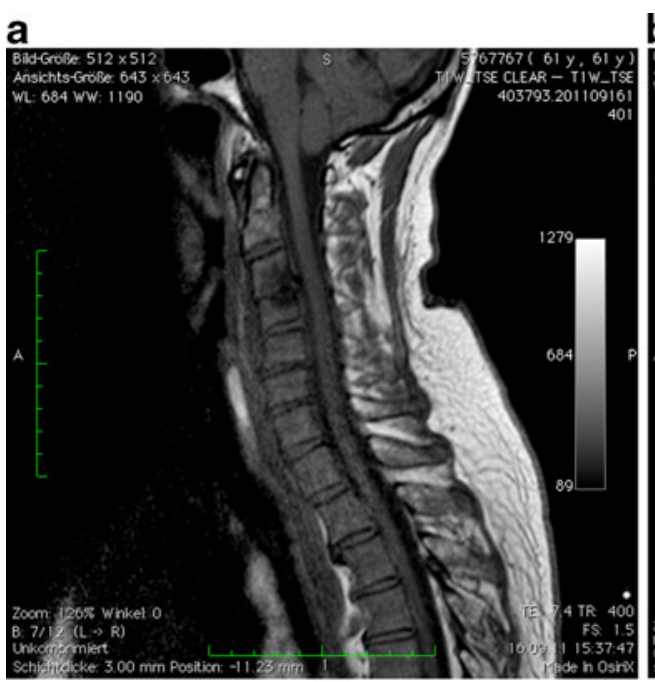

b
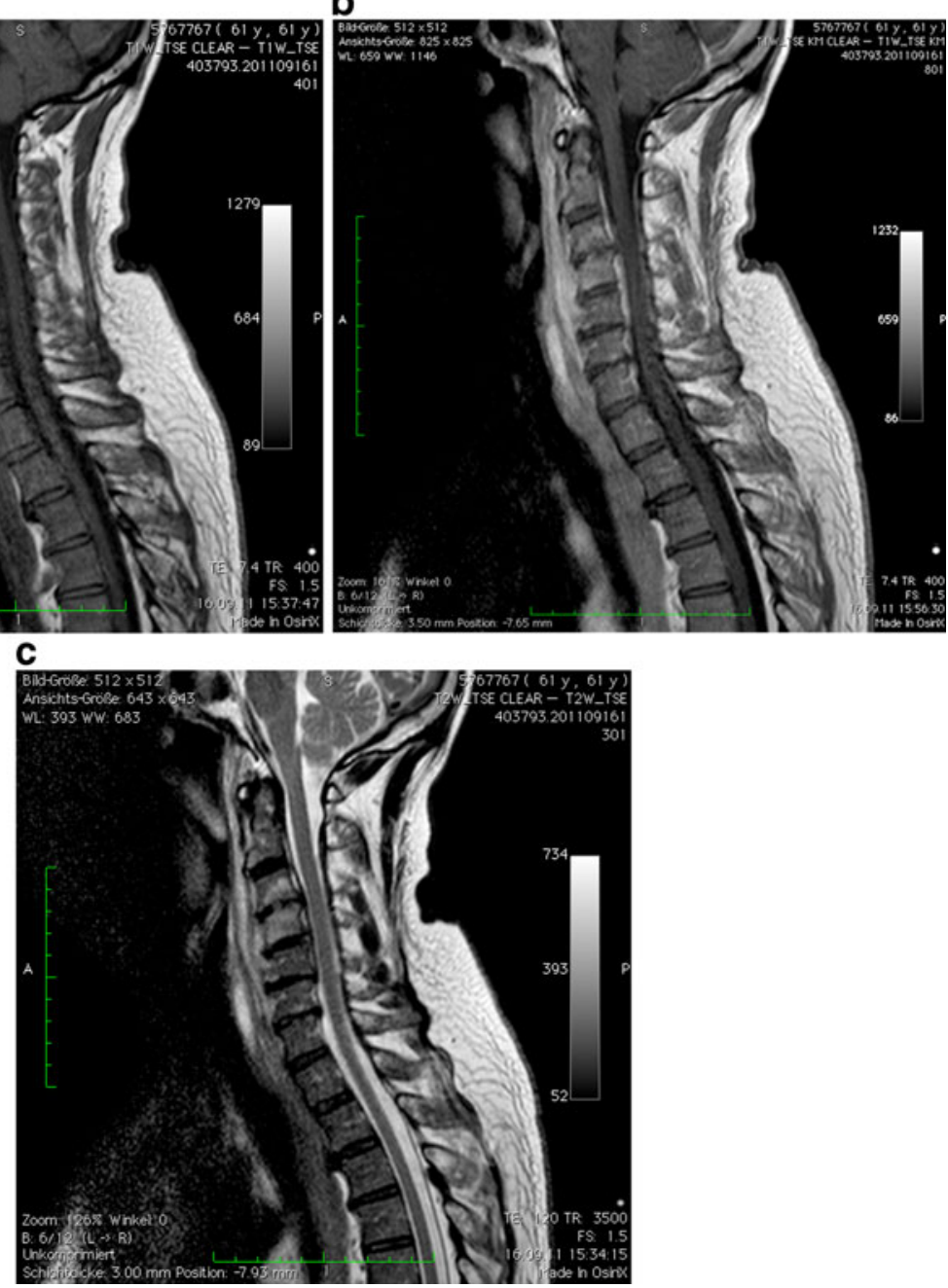

horacoscopy. The advantages of the minimally-invasive thoracoscopic approach are reduced postoperative pain, improved shoulder function, improved postoperative lung function, earlier patient mobilisation, decreased tissue trauma from the approach, better cosmetic results, and shorter hospitalisation time [41]. By splitting the diaphragm, the thoracoscopic approach can be extended inferiorly as far as L2 [41]. In monosegmental defects, fusion can be achieved by interposition of a structural autologous bone graft (i.e. tri-cortical bone graft). In multisegmental disease, an autologous fibula or rib graft could also be used. In case of insufficient anterior stability, supplemental posterior stabilisation should be performed [3].

In the thoracolumbar and lumbar spine, if anterior debridement is not urgently required, posterior stabilisation should be performed initially. In cases of minimal destruction anteriorly, or where sufficient stability is provided by posterior instrumentation and there is increased surgical risk, it might be better to suspend two-stage anterior instrumentation with bone graft interposition. After stabilisation and while
Fig. 3 Postoperative anteroposterior (a) and lateral (b) radiographs after discectomy and debridement of $\mathrm{C} 3 / 4$ and $\mathrm{C} 4 / 5$ and anterior fixation with locking plate $\mathrm{C} 3-\mathrm{C} 5$ 
receiving antibiotic therapy, spontaneous healing of the infected focus with fusion of the adjacent vertebrae can occur over the postoperative course [2]. In cases of monosegmental spondylodiscitis with minor kyphosis, anterior fusion with bone graft alone might be considered [3]. By using anterior stable locking implants, sufficient stabilisation can be achieved [21,29]. Lee et al. published a retrospective series of 18 patients with spondylodiscitis and slight bony destruction, treated with autologous iliac crest bone graft using the PLIF (posterior lumbar interbody fusion) technique [37]. The advantage of a single anterior or posterior approach compared to a combined strategy lies in the lower grade of invasiveness and decreased blood loss, although anterior instrumentation especially at the lumbar spine or lumbosacral junction levels carries increased risks of injuring the large abdominal or pelvic vessels that are often adherent due to inflammation [2]. In a prospective randomised study, Linhardt et al. compared long-term clinical and radiological outcomes after anterior-posterior versus solely anteriorly instrumented thoracic and lumbar fusions performed on 22 patients with spondylodiscitis [21]. For both methods, a fusion rate of $100 \%$ was reached. The mean sagittal loss of correction was $2^{\circ}$ for anterior-posterior fusion, $4^{\circ}$ for a purely anterior fusion at the thoracic level, and $3^{\circ}$ for anterior fusion alone at the lumbar level. Analysis of the SF-36, Oswestry low-back pain disability questionnaire, and visual pain scales showed significantly better results after anterior stabilisation alone for the follow-up periods of two years and 5.4 years after surgery. Similar findings were reported by our group comparing primary stable anterior instrumentation $(n=23)$ with posterior-anterior spinal fusion $(n=$ 32 ). While both groups featured the same correction loss of $2.8^{\circ}$, the time of operation was $50 \%$ longer, and there was a $50 \%$ increase in blood loss for the patients treated with posterior-anterior fusion versus the control group. Bony fusion was achieved in all patients [30]. Known complications of the anterior approach, apart from bleeding, are neurological complications, injury of the internal organs, intestines and ureter, and adhesion formation $[2,29,30]$.

Purely dorsal stabilisation of the thoracolumbar and lumbar spine segments produces load shifts from the anterior spine onto the posteriorly-situated implant. In cases of larger substantive defects or destruction from infection, a bending load could develop, resulting in kyphosis and, in extreme cases, material fatigue [42]. Therefore, additional anterior stabilisation is indicated primarily when larger anterior substance defects are present, as a $360^{\circ}$ fusion at the thoracolumbar and lumbar levels is the most mechanically stable form of instrumentation, with the least risk of pseudarthrosis or kyphosis $[3,4,10]$.

In a retrospective study, Lerner et al. evaluated the clinical and radiological results of single-stage posterior- anterior stabilisation. The anterior substance defect was bridged using bone graft interposition in 42 cases, and titanium cage in 20 cases. Segmental correction loss after cage insertion was significantly lower than that after bone graft interposition $\left(1^{\circ}\right.$ vs. $\left.4.1^{\circ}\right)$. In all cases, bony fusion was achieved [4].

\section{Prognosis}

Spondylodiscitis is potentially life-threatening because it is often not recognised at an early stage. Moreover, it primarily affects elderly patients in reduced states of health or with the accompanying risk factors mentioned above. Mean hospital stay has been reported as 30-49 days, and hospital mortality as $2-17 \%[2,7,21,36,43]$. With our own patients, mean hospital stay is four weeks with a hospital mortality of $2 \%$.

In a retrospective study, Woertgen et al. researched neurological results and quality of life (SF-36) for 62 spondylodiscitis patients after 16.4 months. Of these patients, $45 \%$ had been treated conservatively and $55 \%$ surgically. For patients with neurological deficits present prior to surgery, motor deficits persisted in $30 \%$, and hypaesthesia in $90 \%$. In addition, quality of life for all patients was far lower than that of a normal population. Slightly improved quality of life and significantly higher levels of patient satisfaction were found in the surgicallytreated patients [43]. In a group of 25 spondylodiscitis patients, Lerner et al. identified an improvement of neurological deficits for $76 \%$ after a mean of 2.6 years, while $20 \%$ showed no change. In $75 \%$ of patients with acute paraplegia, ambulation was restored after therapy [4]. Similar results can be found in other published studies $[2,3$, 38]. Overall, a relapse of spondylodiscitis is unlikely to occur. In the literature, relapse rates have been recorded as $0-7 \%[2,3,21,29,38,41]$.

\section{References}

1. Butler JS, Shelly MJ, Timlin M, Powderly WG, O'Byrne JM (2006) Nontuberculous pyogenic spinal infection in adults: a 12-year experience from a tertiary referral center. Spine 31(23):2695-2700

2. Frangen TM, Kalicke T, Gottwald M, Andereya S, Andress HJ, Russe OJ, Muller EJ, Muhr G, Schinkel C (2006) Surgical management of spondylodiscitis. An analysis of 78 cases. Unfallchirurg 109(9):743-753

3. Klockner C, Valencia R, Weber U (2001) Alignment of the sagittal profile after surgical therapy of nonspecific destructive spondylodiscitis: ventral or ventrodorsal method - a comparison of outcomes. Orthopade 30(12):965-976

4. Lerner T, Hackenberg L, Rosler S, Joosten U, Halm H, Liljenqvist U (2005) Surgical therapy of unspecific and specific Spondylodiscitis. Z Orthop Ihre Grenzgeb 143(2):204-212 
5. Muller EJ, Russe OJ, Muhr G (2004) Osteomyelitis of the spine. Orthopade 33(3):305-315

6. Nolla JM, Ariza J, Gomez-Vaquero C, Fiter J, Bermejo J, Valverde J, Escofet DR, Gudiol F (2002) Spontaneous pyogenic vertebral osteomyelitis in nondrug users. Seminars Arthritis Rheum 31(4):271-278

7. Schinkel C, Gottwald M, Andress HJ (2003) Surgical treatment of spondylodiscitis. Surg Infect 4(4):387-391

8. Tsiodras S, Falagas ME (2006) Clinical assessment and medical treatment of spine infections. Clin Orthop Relat Res 444:38-50

9. Acosta FL Jr, Chin CT, Quinones-Hinojosa A, Ames CP, Weinstein PR, Chou D (2004) Diagnosis and management of adult pyogenic osteomyelitis of the cervical spine. Neurosurg Focus 17(6):E2

10. Cramer J, Haase N, Behre I, Ostermann PAW (2003) Spondylitis und Spondylodiszitis. Trauma und Berufskrankheit (5)

11. Eysel P, Peters K (1997) Spondylodiszitis. In: Peters K, Klosterhalfen B (eds) Bakterielle Infektionen der Knochen und Gelenke. Enke, Stuttgart, pp 52-93

12. Sobottke R, Seifert H, Fatkenheuer G, Schmidt M, Gossmann A, Eysel P (2008) Current diagnosis and treatment of spondylodiscitis. Dtsch Arztebl Int 105(10):181-187

13. Gouliouris T, Aliyu SH, Brown NM (2010) Spondylodiscitis: update on diagnosis and management. J Antimicrob Chemother 65(Suppl 3):ii11-ii24

14. Grados F, Lescure FX, Senneville E, Flipo RM, Schmit JL, Fardellone P (2007) Suggestions for managing pyogenic (nontuberculous) discitis in adults. Joint Bone Spine 74(2):133-139

15. Zarghooni K, Rollinghoff M, Siewe J, Fatkenheuer G, Seifert H, Eysel P, Sobottke R (2010) Spondylodiscitis - an interdisciplinary challenge. Dtsch Med Wochenschr 135(23):1182-1185

16. Eismont FJ, Bohlman HH, Soni PL, Goldberg VM, Freehafer AA (1983) Pyogenic and fungal vertebral osteomyelitis with paralysis. J Bone Joint Surg Am 65(1):19-29

17. Sapico FL, Montgomerie JZ (1990) Vertebral osteomyelitis. Infect Dis Clin North Am 4(3):539-550

18. Lew DP, Waldvogel FA (2004) Osteomyelitis. Lancet 364 (9431):369-379

19. Shuford JA, Steckelberg JM (2003) Role of oral antimicrobial therapy in the management of osteomyelitis. Curr Opin Infect Dis 16(6):515-519

20. Jensen AG, Espersen F, Skinhoj P, Frimodt-Moller N (1998) Bacteremic Staphylococcus aureus spondylitis. Arch Intern Med 158(5):509-517

21. Linhardt O, Matussek J, Refior HJ, Krodel A (2007) Long-term results of ventro-dorsal versus ventral instrumentation fusion in the treatment of spondylitis. Int Orthop 31(1):113-119

22. Hoffner SE (1994) Pulmonary infections caused by less frequently encountered slow-growing environmental mycobacteria. Eur J Clin Microbiol Infect Dis 13(11):937-941

23. Manfredi R, Nanetti A, Morelli S, Ferri M, Valentini R, Calza L, Chiodo F (2004) A decade surveillance study of Mycobacterium xenopi disease and antimicrobial susceptibility levels in a reference teaching hospital of northern Italy: HIV-associated versus non-HIV-associated infection. HIV Clin Trials 5(4):206215

24. Medical Section of the American Lung Association (1997) Diagnosis and treatment of disease caused by nontuberculous mycobacteria. This official statement of the American Thoracic Society was approved by the Board of Directors, March 1997. Am J Respir Crit Care Med 156(2 Pt 2):S1-S25

25. Kim CW, Perry A, Currier B, Yaszemski M, Garfin SR (2006) Fungal infections of the spine. Clin Orthop Relat Res 444:92-99
26. van Ooij A, Beckers JM, Herpers MJ, Walenkamp GH (2000) Surgical treatment of aspergillus spondylodiscitis. Eur Spine J 9 (1):75-79

27. Sobottke R, Rollinghoff M, Zarghooni K, Schluter-Brust K, Delank KS, Seifert H, Zweig T, Eysel P (2010) Spondylodiscitis in the elderly patient: clinical mid-term results and quality of life. Arch Orthop Trauma Surg 130(9):1083-1091

28. Flamme CH, Frischalowski T, Gosse F (2000) Possibilities and limits of conservative therapy of spondylitis and spondylodiscitis. Z Rheumatol 59(4):233-239

29. Eysel P, Hopf C, Meurer A (1994) Korrektur und Stabilisierung der infektbedingten Wirbelsäulendeformität. Orthop Prax 11:696703

30. Eysel P, Hopf C, Vogel I, Rompe JD (1997) Primary stable anterior instrumentation or dorsoventral spondylodesis in spondylodiscitis? Results of a comparative study. Eur Spine J 6(3):152157

31. Hodgson AR, Stock FE (2006) The Classic: Anterior spinal fusion: a preliminary communication on the radical treatment of Pott's disease and Pott's paraplegia. 1956. Clin Orthop Relat Res 444:10-15

32. Krodel A, Sturz H (1989) Differentiated surgical and conservative treatment of spondylitis and spondylodiscitis. Z Orthop Ihre Grenzgeb 127(5):587-596

33. Alangaden GJ, Lerner SA (1997) The clinical use of fluoroquinolones for the treatment of mycobacterial diseases. Clin Infect Dis 25(5):1213-1221

34. Osenbach RK, Hitchon PW, Menezes AH (1990) Diagnosis and management of pyogenic vertebral osteomyelitis in adults. Surg Neurol 33(4):266-275

35. Oga M, Arizono T, Takasita M, Sugioka Y (1993) Evaluation of the risk of instrumentation as a foreign body in spinal tuberculosis. Clinical and biologic study. Spine (Phila Pa 1976) 18 (13): 1890-1894

36. Isenberg J, Jubel A, Hahn U, Seifert H, Prokop A (2005) Multistep surgery for spondylosyndesis. Treatment concept of destructive spondylodiscitis in patients with reduced general condition. Orthopade 34(2):159-166

37. Lee JS, Suh KT (2006) Posterior lumbar interbody fusion with an autogenous iliac crest bone graft in the treatment of pyogenic spondylodiscitis. J Bone Joint Surg Br 88(6):765-770

38. Heyde CE, Boehm H, El Saghir H, Tschoke SK, Kayser R (2006) Surgical treatment of spondylodiscitis in the cervical spine: a minimum 2-year follow-up. Eur Spine J 15(9):1380-1387

39. An HS, Singh K, Vaccaro AR, Wang G, Yoshida H, Eck J, McGrady L, Lim TH (2004) Biomechanical evaluation of contemporary posterior spinal internal fixation configurations in an unstable burst-fracture calf spine model: special references of hook configurations and pedicle screws. Spine (Phila Pa 1976) 29 (3):257-262

40. Hazelrigg SR, Cetindag IB, Fullerton J (2002) Acute and chronic pain syndromes after thoracic surgery. Surg Clin North Am 82 (4):849-865

41. Muckley T, Schutz T, Schmidt MH, Potulski M, Buhren V, Beisse $R$ (2004) The role of thoracoscopic spinal surgery in the management of pyogenic vertebral osteomyelitis. Spine (Phila Pa 1976) 29(11):E227-E233

42. Delamarter RB, Sherman J, Carr JB (1995) Pathophysiology of spinal cord injury. Recovery after immediate and delayed decompression. J Bone Joint Surg Am 77(7):1042-1049

43. Woertgen C, Rothoerl RD, Englert C, Neumann C (2006) Pyogenic spinal infections and outcome according to the 36-item short form health survey. J Neurosurg 4(6):441-446 